Yrysov Keneshbek Bakirbaevich, holder of post-doctoral degree in Medicine, professor, assistant principal of state language and education of I. K. Akhunbaev Kyrgyz State Medical Academy

E-mail:dr.ysi@mail.ru

Ydrysov Ismatilla Toktosunovich, holder of doctorate degree in Medicine, assistant professor, head of a surgery department of postgraduate medical education of Osh state university

Kalyev Kursanbek Muktarovich, holder of doctorate degree in Medicine, teaching assistant of traumatology department of medicine faculty of Osh state university

\title{
ANALYSIS OF MORTALITY CASES FROM CONCOMITANT INJURIES: EXTRACRANIAL INJURIES COMBINED WITH CRANIOCEREBRAL INJURIES
}

\begin{abstract}
Concomitant injury is the mechanical damage of two or more organs of different cavities or simultaneous damage of the internal organ (or organs) and the musculoskeletal system (single or multiple). Concomitant injury is defied by two or more injuries, which require the help of different medical specialists (neurosurgeon, traumatologist, abdominal surgeon, oculist, maxillofacial surgeon, etc.). CCCI, which occurs in $43 \%$ of patients with all kinds of injuries, deserve special attention. Mortality among these patients ranges from $28.6 \%$ to $30.7 \%$ [4; $5 ; 6$; .

Analysis of the treatment's result used in this study shows that out of 251 patients, 46 died after hospitalization. The overall mortality rate was $18.3 \%$. Depending on the types of injuries in combination with extracranial injury, the mortality rates were as follows: in combination with damage to the maxillofacial region, 3 cases $(6.5 \%)$, chest and thoracic injuries - 14 (30.4\%), abdominal cavity organs damage - 11 (23.9\%) and multiple extracranial injuries - 18 (39.1\%).

Researchers all over the world continue to improve the diagnostic techniques and treatment of combined extracranial injuries, aiming to find better means of treatment and to improve its quality and efficient use of available resources $[7 ; 8]$.

Keywords: severe concomitant craniocerebral injury, injury of thoracic organs and organs of abdominal cavity, analysis of mortality cases from extracranial injuries.

Rationale. Concomitant craniocerebral injury (CCCI) is the most common type among all concomitant injuries. Those injuries make up from 43 to $68 \%$ cases and most predominate are craniocerebral injuries. Concomitant injuries are more than combination

of various organs' injuries, they can be considered as a special category of injuries, where pathological process has its own peculiarities and laws $[1 ; 2 ; 3]$.

General characteristics of patients and research techniques. This research has a detailed
\end{abstract}


analysis of the complex assessments and the results of treatment of 251 patients with severe craniocerebral injuries, combined with extracranial injuries of different localization in the acute stage, as the basis. The patients treated were from Osh Interregional Clinical Hospital, Osh City Clinical Hospital, Nookat Subdistrict Hospital, Jalal-Abad Regional Hospital and Kyzyl-Kiya Sub-district Hospital in 2013-2016.

Out of 251 patients, 46 died after hospitalization, the overall mortality in the present research was $18.3 \%$. A number of factors were found significant in relation to the probability of mortality. High mortality rates were determined in cases of patients older than 61 years, whereas the sex of patients was found not significant to the mortality probability. In our research, there were 201 (80.1\%) males and 50 (19.9\%) females $(p<0.05)$. The average age of patients ranged from 13 to 86 years and was $45.2 \pm 7.6$ years.

Out of 65 people who were hospitalized in the state of shock or terminal state, 32(49.2\%) died.

The main causes of death in case of extracranial injuries were:

1) severe craniocerebral injury combined with severe extracranial injuries -16 patients $(34.8 \%)$;

2 ) diagnostic errors -5 cases (10.9\%), including intracranial injuries (hematomas) in 3 patients, $1-$ liver rupture and 1 - spleen rupture;

3) secondary complications - 14 patients (30.4\%) including inflammatory - 12 (pneumonia-9, peritonitis-2, meningitis-1), thromboembolism of the pulmonary artery-2. Total mortality rate with compression of the brain in patients with combined craniocerebral injury and shock was $70.8 \%$, and postoperative mortality $-63.0 \%$.

Given the long-term anchorage of the patients in the position on the back during reanimation and anti-shock treatment, preoccupation of the surgical team with the implementation of urgent anti-shock measures, such as surgical interventions, the implementation of some techniques of treating severe craniocerebral injury becomes difficult or impossible $[8 ; 9 ; 10]$. We have developed some treatment tech- niques, the use of which is possible in these conditions. It includes techniques of intensive sanitation of subarachnoid spaces and the removal of intracranial hematomas through mini burr holes.

Treatment of chest injuries was also dependent on the predictors of severity and outcome of injury. All modern types of conservative and surgical treatment were used, except for thoracobrachial dressings, which, because of the complexity of application, bulkiness, difficulties associated with examining and caring for the patients, were not used. Treatment of patients with injuries of the chest, which have had a favorable prognosis and duration of shock up to 8 hours, was performed as early as possible, using all available techniques of surgery and conservative treatment.

We propose a technique for treating injuries of the thorax and ribs in a concomitant craniocerebral injury. To perform this technique, $1 \mathrm{ml}$ of a $2 \%$ solution of lidocaine should be slowly injected intradermally to the patient. The administration should be performed below the xiphoid process once a day for 3-5 days. This technique allows the provision of a steady decrease in the pain syndrome of the patients with chest injuries and fracture of the ribs in a concomitant craniocerebral injury with a minimal expenditure of analgesics due to a decrease in the activity of the sympathetic nervous system.

This technique increases the effectiveness of treatment, shortens the recovery period and reduces disability rate among patients with concomitant craniocerebral injuries.

The proposed technique allowed us to achieve a qualitative improvement in the results of treatment of patients with concomitant craniocerebral injuries, reduce the incidence of disability, and prevent possible complications, both in the acute period of severe craniocerebral injury and in the postoperative period. This technique was used in the diagnostic and treatment of 15 patients. After the treatment, using this treatment technique, all patients were discharged in a satisfactory condition. 
Practical use of the presented technique with the introduction to the therapeutic and diagnostic tactics, based on the objective indicators of the severity of the condition of the patients, allowed to improve the quality and effectiveness of treatment of one of the most severe categories of patients - the patients with a concomitant craniocerebral injury, which suffered from shock. So, the mortality rate of patients with severe cerebral contusion decreased by $5.4 \%$ and with compression of the brain by $2.2 \%$. The mortality in the complicated injuries has decreased by $6.8 \%$ (from 48.5 to $41.7 \%$ ). The overall mortality rate decreased by $18.3 \%$, and the mortality in the shock period was $10 \%$ compared lower to the mortality in a similar group of patients treated according to usual schemes.

The highest mortality was observed in the presence of compression of the brain and in the combination of severe craniocerebral injury with multiple thoracoabdominal injuries. It was noted that the lethal outcome was more often observed in the I group of patients with concomitant craniocerebral injury in the first and second periods of wound dystrophy (respectively $81.6 \%$ and $63.3 \%$ ) and was mainly due to shock, acute blood loss, and the development of early pneumonia and thromboembolic complications.

Clinical and morphological comparisons of 46 deceased patients showed a lack of parallelism between clinical manifestations of injury and brain injuries of the patients. Contusions of the brain differed in multiplicity, depth and expressed hemorrhagic component, perifocal edema was expressed slightly.

Outcomes of a concomitant craniocerebral injuries depended on the severity and concomitant factor of this type of injury, the presence of shock, blood loss, alcohol intoxication, the dynamics of impaired consciousness, the types and the mechanism of injuries, the speed, volume, and adequacy of the provided treatment up to the hospital stage, the terms of hospitalization, the time of the beginning and completeness of the treatment, complications and accompanying diseases. Prediction of immediate outcomes and early complications of concomitant injury required a comprehensive development of approaches taking into account all the necessary clinical and paraclinical data and information on the age aspect.

To determine our strategy and surgical treatment of intracranial hematomas, we analyzed the causes of death of operated patients to retrospectively evaluate in what cases with modern neurosurgery and resuscitation facilities, the intervention will be ineffective, and in what cases, with more correct and timely treatment, one could hope for benign outcome. Patients of young and middle age group and elderly (people of 60 years and older) were studied by us in terms of the causes that led to death.

The mortality rate prevailed among the patients with a bilateral injury of the midbrain ( 3 cases) and bilateral brain bridge ( 8 cases), and duration of coma was noted in 4(1-16) \pm 2.4 cases with a supratentorial lesions, in $8(3-17) \pm 2.8$ - with damage to the brainstem and in $14(2-24) \pm 6.1$ - bilateral injury of the midbrain.

In the group of patients of 21-40 years old, out of 73 patients, 3 patients died. When analyzing the causes of mortality, it was revealed that 1 of them died in the first hours or days, from a bruise-hemorrhage to the brainstem, 2 patients - in the postoperative period from cerebral edema with an incision that developed suddenly on day 3-6 after the removal of intracranial hematoma. At first, the condition of these patients improved, they became available to the contact, but then, within a few hours, a cerebral symptomatology developed followed by addition of the stem symptomatology and termination of the vital functions. On pathoanatomical dissection, these patients showed a sharp edema and swelling of the brain, which led to compression of the brainstem, patients died due to hemodynamic disorders in the brain.

Results. Therefore, 3 patients from this group died, because of the reasons that made treatment ineffective. Removal of foci of crushing, intensive anti-edematous therapy could rescue the patients. 
In the middle age group (41-60 years), 13 patients died. In this group, on the background of the hypertensive disease, atherosclerosis and chronic diseases present in patients, the severity extends beyond cerebral complications in the form of hypostatic pneumonia, fibro-purulent tracheobronchitis, and hemorrhages to the parenchymal organs. In this group, the frequency of damage to the bones of the skull and internal organs was high, 1 patient of this group died in the postoperative period during the first day of primary stem hemorrhage. This patient, except for the main focus and hemorrhages in the hemisphere and the brain stem, had small hemorrhages in the stomach, pericardium, lungs, intestines and adrenal glands. This combination of hemorrhages should be considered as a vasomotor or diapedesis. Hemorrhages in the adrenal glands, as well as hemorrhages in the stem are considered incompatible with life. The dependence of the frequency of combined hemorrhages in the parenchymal organs on age was noted. Their frequency increases with age. In all cases of hemorrhage, edema-swelling of the brain developed with infringement in the occipital foramen of the brain stem sections. Such compression of the stem leads to its ischemia with violation of cardiac and respiratory functions.

Thus, it was not possible to save one patient with a hemorrhage in the brain stem, despite the use of existing techniques and means.

Among the elderly group (61 years and older), 15 patients died, 2 died on the first day due to the increasing stem decompensation.

Two patients of this group died of hemorrhagic stroke on the background of the developed hyper- tensive crisis. Their condition after the operation improved, but then on day 2-3 they died as a result of rapidly developing vital disturbances. The biopsy has shown that fresh hemorrhages were found in the brain tissue. The remaining 2 patients died on the $5-14^{\text {th }}$ day with gradual deterioration of the condition. A few hours before death, the condition deteriorated sharply, followed by a loss of consciousness and a syndrome of "floating" eyeballs, tonic convulsions and termination of vital functions, which led to death. Autopsy has shown areas of traumatic softening of the cortex and white matter of the brain, secondary hemorrhage in the brainstem, more often found in the bridge. Secondary hemorrhages were associated, evidently, with the growing pathological vascular reactions that led to hemorrhage into the brain stem. All had hypostatic pneumonia of varying severity, atherosclerotic changes in blood vessels, in particular cerebral vessels.

Out of 46 (18.3\%) of the deceased patients, 6 developed primary hemorrhages of the stem, which were the main cause of death of the patients. The severity of the injury in these patients did not give any real reason to believe that any of them could be saved. Other 5 patients had different causes of death. Here, death was due to secondary reactions to injury. The causes of such violent reactions that led to death were the centers of extensive traumatic brain lesions.

Conclusion. Therefore, we can note that as the age of the patients we observe increases, the frequency of secondary stem hemorrhages, pathological vascular reactions of the brain, hypostatic pneumonia increases on the background of already existing cardiovascular and pulmonary pathologies.

\section{References:}

1. Bondarenko A.V. Hospital lethality with combined trauma and the possibility of its reduction [Text] / A. V. Bondarenko, V.A. Pelleganchuk, O.A. Gerasimova // Vestn. traumatol. and orthopedist., 2006.No. 3.- P. 49-52.

2. Wagner E. A. Surgical tactics in severe combined trauma of the chest in the early period of traumatic disease (Diagnosis, treatment, outcomes) [Text] / E.A. Wagner, V.A. Bruns // Vestn. traumatol. and orthopedics. N. N. Pirogova, 1998.- No. 2.- P. 3-7. 
3. Verkhovsky A. I. Combined mechanical trauma. Combined craniocerebral injury: A teaching method, a manual [Text] / A. I. Verkhovsky, I. V. Kurshakova / Institute of First Aid to them. II Dzhanelidze,St. Petersburg, 2007.- Issue. 19.- 59 p.

4. Grinev M.V. Combined trauma: the essence of the problem, the ways of solution [Text] / M. V. Grinev // Assist in case of combined trauma.-St. Petersburg, 2002.- P. 58-63.

5. Kachkov I.A. Epidemiology of severe combined craniocerebral trauma and organisation of medical care for victims in the Moscow Region [Text] / I. A. Kachkov, B.A. Kocherezhkin, V. Schmelev // Neurosurgery, 2007.- No. 4.- P. 56-59.

6. Kondakov E. N. Craniocerebral trauma: A guide for physicians in non-specialized hospitals [Text] / E. N. Kondakov, V.V. Krivetsky // - St. Petersburg,-SpetsLit., 2002.- 186 s.

7. Konovalov A.N. Clinical manual on craniocerebral trauma: in 2 tons. [Text] / A. N. Konovalov, JI. B. Likhterman, A. A. Potapov // - M: Antidor, 2002.- T. 1.- 550 p.

8. Mamytov M.M. Neurosurgical problems of focal lesions of the brain [Text] / M. M. Mamytov, K. B. Yrysov, E. M. Mamytova // - Bishkek: Altyn tamga, 2010.- 190 s.

9. Becker C.D. The trauma concept: the role of MDCT in the diagnosis and management of visceral injuries [Text] / C.D. Becker, P. A. Poletti // Eur Radiol., 2015.- Vol. 15.- P. 105-109.

10. Gennarelli T. A. Mortality of patients with head injury and extracranial injury treated in trauma centers [Text] / T.A. Gennarelli, H.R. Champion, W. M. Alves // J Trauma, 2016.-Vol. 29.-P. 1193-1201. 